curves were generated and the area under the curve (AUC) calculated.

Results At baseline, the plasma NGAL $(69.4 \pm 29.8 \mathrm{ng} / \mathrm{ml})$ was correlated positively with creatinine $(90.9 \pm 20.4 \mu \mathrm{mol} / \mathrm{L})\left(\mathrm{r}^{2}=0.341\right.$, $\mathrm{p}<0.001)$ The plasma NGAL increased at $2 \mathrm{~h}$ and reached peak at $8 \mathrm{~h}$ and decreased at $24 \mathrm{~h}$ after procedures. Among all the patients, 29 patients (12.6\%) developed CIAKI. The sensitivity, specificity, and ROC curve for prediction of CIN were excellent for the $2 \mathrm{~h}$ plasma NGAL $(75 \%, 89 \%$, and 0.90 , respectively).

Conclusion NGAL seems to be a potential early biomarker for CIAKI.

\section{e0514 THE SAFETY AND FEASIBILITY OF REPEATED PERCUTANEOUS TRANSRADIAL CORONARY INTERVENTION IN THE SAME ROUTE}

doi:10.1136/hrt.2010.208967.514

Nie Bin, Zhou Yujie, Yang Qing, Cheng Wan-Jun, Wang Zhi-Jian, Wang Jian-Long. Department of Cardiology, Beijing Anzhen Hospital, Capital Medical University, Beijing, China

Background The radial approach has been increasingly used as an alternative to femoral access. And more procedures of repeated transradial coronary intervention (r-TRI) are performed. Few data about r-TRI has been obtained. Therefore, we tried to investigate the safety and feasibility of repeated transradial coronary intervention $(\mathrm{r}-\mathrm{TRI})$ in the same route.

Methods A total of 423 consecutive eligible patients undergoing repeated TRI were enrolled in r-TRI group, and 846 patients with initial TRI were assigned to i-TRI group in a 2- to- 1 ratio matched by ages and gender. The primary endpoint included the success rate of procedure and incidence of vascular related complications.

Results The baseline clinical characteristics in two groups were comparable. The success rate of procedure in r-TRI and i-TRI was similar $(96.0 \%$ vs $97.5 \%, p=0.130)$. In subgroup analysis (coronary angiography only or angiography with percutaneous coronary intervention), the similar result was also observed. The puncture numbers and incidence of radial artery spasm in r-TRI group were significant higher than $\mathrm{i}-\mathrm{TRI}$ group $(\mathrm{p}=0.034$ and $\mathrm{p}<0.001$, respectively). The other procedural outcomes in two groups were identical. With respect to the incidence of overall vascular related complication and independent event, there were no significant difference in spite of higher incidence in r-TRI group $(1.2 \%$ vs $0.7 \%$, $\mathrm{p}=0.521)$. The patients in $\mathrm{i}-\mathrm{TRI}$ group had more comfortable feeling than $r$-TRI group $(p<0.001)$.

Conclusions Repeated TRI produces an equal success rate and a lower vascular complication when compared to initial TRI. It should be considered as an acceptable and safe procedure.

\section{e0515 THE VALUE OF EXAMINATION OF FOREARM ARTERY USING DOPPLER ULTRASOUND BEFORE TRANS}

doi:10.1136/hrt.2010.208967.515

Nie Bin, Zhou Yujie, Yang Qing, Cheng Wan-Jun, Wang Zhi-Jian. Department of Cardiology, Beijing Anzhen Hospital, Capital Medical University, Beijing, China

Objective To explore the value of examination of fore arm artery using Doppler ultrasound before transradial coronary intervention (TRI).

Methods Consecutive patients undergoing elective TRI were screened. Including criteriors were as follows: positive Allen's test result of right radial artery (RRA). The primary endpoints included the procedural success rate and incidence of vascular related complications. The secondary endpoints were: the average diameter of fore arm diameter and anatomic variations of approach.

Results A total of 1863 patients were eligible and assigned to ultrasound group (931 cases) and convention group without ultrasound examination (932 cases). The baseline clinic characteristics in two groups were comparable. 181 cases of anatomic variation in RRA approach were detected before procedure. Among these, approach in 13 cases changed to other routes from RRA. The procedural success rate in ultrasound group and convention group was similar ( $98.0 \%$ vs $97.2 \%, p=0.292)$. The incidence of vascular complications in ultrasound group was significantly lower than convention group $(8.7 \%$ vs $11.6 \%, p=0.039)$. Regarding artery perforation and radial artery occlusion, the incidences of those in ultrasound group occurred less frequently $(p=0.025$ and 0.028 , respectively).

Conclusions Examination of fore arm artery using Doppler ultrasound could not only detect anatomic variations and reduce vascular complication effectively, but also be helpful to selective suitable approach and instruments.

\section{e0516 SAFETY AND EFFICACY OF TRANSULNAR APPROACH FOR CORONARY ANGIOGRAPHY AND INTERVENTION}

doi:10.1136/hrt.2010.208967.516

Li Yunzhi, Zhou Yujie. Department of Cardiology, Beijing Anzhen Hospital, Capital Medical University, Beijing, China

Background Transradial approach, which is now widely used in coronary angiography and intervention may be advantageous with respect to the femoral access due to the lower incidence of vascular complications. Transulnar approach has been proposed for elective procedures in patients not suitable for transradial approach.

Objective The objective of this study was to evaluate the safety and efficacy of the transulnar approach vs the transradial approach for coronary angiography and intervention.

Methods 240 patients undergoing coronary angiography, followed or not by intervention, were randomised to transulnar (TUA) or transradial approach (TRA). Doppler ultrasound assessments of the forearm vessels were scheduled for all patients before procedures, 1 day and 30 days after procedures. The primary end point was access site vascular complications during hospitalisation and 30 day follow-up. MACE as secondary end point was recorded till 30 day follow-up.

Results Successful puncture was achieved in 98.3\% (118/120) of patients in the TUA group, and in 100\% (120/120) of patients in the TRA group. Coronary angiographies were performed in 40 and 39 patients in TUA and TRA group. Intervention procedures were performed in 78 and 83 patients in TUA and TRA group, respectively. The incidence of artery stenosis 1 day and 30 day after procedures was $11 \%$ vs $12.3 \%$ and $5.1 \%$ vs $6.6 \%$ in TUA and TRA group, respectively. Asymptomatic access site artery occlusion occurred in $5.1 \%$ vs $1.7 \%$ of patients 1 day and 30 day after transulnar angioplasty, and in $6.6 \%$ vs $4.9 \%$ of patients 1 day and 30 day after transradial angioplasty. Minor bleeding was still observed at the moment of the ultrasound assessment in $5.9 \%$ and $5.7 \%$ of patients in TUA and TRA group, respectively $(p=0.949)$. No big forearm haematoma, and A-V fistula were observed in both groups. Freedom from MACE at 30 day follow-up was observed in all patients.

Conclusion The transulnar approach is as safe and effective as the transradial approach for coronary angiography and intervention. It is an attractive opinion for experienced operators who are skilled in this technique, particularly in cases of anatomic variations of the radial artery, radial artery small-calibre or thin radial pulse. 